\title{
NOTABREVE
}

\section{BIODIVERSIDAD CAPRINA EN ESPAÑA}

\author{
GOATBIODIVERSITYINSPAIN
}

\author{
Martínez, A.M. ${ }^{1 *}$, Landi, V. ${ }^{1}$, Amills, M. ${ }^{2}$, Capote, J. ${ }^{3}$, Gómez, M. ${ }^{4}$, Jordana, J. ${ }^{2}$, Ferrando, A. ${ }^{2}$ \\ Manunza, A. ${ }^{2}$, Martín, D. ${ }^{1}$, Pons, A. ${ }^{5}$, Vidal, $0 .{ }^{6}$ y Delgado, J.V. ${ }^{1}$ \\ 1Departamento de Genética. Universidad de Córdoba. Córdoba. España. ib2mamaa@uco.es \\ ${ }^{2}$ Departament de Ciència Animal i dels Aliments. Universitat Autònoma de Barcelona. Bellaterra. \\ España. \\ ${ }^{3}$ Instituto Canario de Investigaciones Agrarias. La Laguna. Tenerife. España. \\ ${ }^{4}$ Servicio de Ganadería. Diputación Foral de Bizkaia. Bilbao. España. \\ ${ }^{5}$ Instituto de Biología Animal de Balears, SA. Son Ferriol. Palma de Mallorca. España. \\ ${ }^{6}$ Departament de Biologia. Universitat de Girona. Girona. España.
}

\section{Palabras claVe adicionales}

Diversidad genética. Microsatélites. Razas autóctonas.

\section{RESUMEN}

La especie caprina se localiza predominantemente en la España seca con gran protagonismo de Andalucía, Castilla-La Mancha, Canarias, Murcia y Extremadura. En este trabajo se analizan 867 muestras de 18 poblaciones caprinas españolas provenientes de diferentes zonas de la Península Ibérica (Azpi-Gorri, Blanca de Rasquera, Blanca Andaluza, Blanca Celtibérica, Florida, Malagueña, Murciano-Granadina, Negra Serrana, Payoya y Retinta) y de los archipiélagos Balear (Ibicenca, Mallorquina y cabra de Formentera) y Canario (Ajuí, Majorera, Palmera, Tinerfeña del Norte y Tinerfeña del Sur). Se amplifican 23 microsatélites de ADN y se determina la variación genética de las razas mediante el análisis del número medio de alelos, la heterocigosis, y el estadístico $F_{\text {IS }}$. Los valores de heterocigosis esperada $\left(\mathrm{H}_{\mathrm{e}}\right)$ oscilan entre 0,524 en la raza Palmera y 0,721 en la Florida mientras que la heterocigosis observada $\left(\mathrm{H}_{0}\right)$ fluctúa entre 0,515 en la Palmera y 0,705 en la Retinta. En España existe una diversidad genética caprina elevada con valores de número medio de alelos, heterocigosis esperada y observada para todas las razas de 7,04, 0,668 y 0,621 respectivamente.

Presentado al Congreso SERGA (2010, Asturias).

\section{AdDitiOnAL KEYWORDS}

Genetic diversity. Microsatellites. Local breeds.

\section{SUMMARY}

Goats are located predominantly in the dry Spain specially in Andalusia, Castilla-La Mancha, the Canary Islands, Murcia and Extremadura. In this paper we have analyzed 867 samples of 18 Spanish goat populations from different parts of the Iberian Peninsula (Azpi-Gorri, Blanca de Rasquera, Blanca Andaluza, Blanca Celtibérica, Florida, Malagueña, Murciano-Granadina, Negra Serrana, Payoya and Retinta) and the Balearic (Ibicenca, Mallorquina and Formentera goat) and Canarian Archipelagos (Ajuí, Majorera, Palmera, Tenerife North and Tenerife South). Twenty-three microsatellites have been amplified and the withinbreed genetic variation has been calculated through the analysis of the mean number of alleles, heterozygosity and $F_{\text {IS }}$ The values of expected heterozygosity $(\mathrm{He})$ per breed ranged from 0.524 to 0.721 in Palmera and Florida, respectively, while the observed heterozygosity $(\mathrm{Ho})$ ranged between 0.515 and 0.705 in Palmera and Retinta, respectively. Goat genetic diversity in Spain is very high, with a mean number of alleles, expected and observed heterozigosities across the breeds of $7.04,0.668$ and 0.621 , respectively.

\section{INTRODUCCIÓN}

El censo de caprinos en España es el 


\section{MARTÍNEZ ETAL.}

segundo de Europa, después de Grecia, y se localiza predominantemente en la España seca con gran protagonismo de Andalucía, Castilla-La Mancha, Canarias, Murcia y Extremadura. Según la última actualización del Catálogo Oficial de Razas de Ganado (RD 2129/2008), en España hay 22 razas caprinas autóctonas reconocidas, 17 de ellas son razas en peligro de extinción y 5 razas de fomento. En España existe una gran riqueza racial siendo la mayor parte de las razas explotadas autóctonas, tratándose de razas con elevada rusticidad, adaptadas a los variados ecosistemas existentes tanto en la península como en los dos archipiélagos. La mayor parte de estas razas están fuertemente ligadas por tanto a su entorno con lazos históricos y culturales. El objetivo de este trabajo es conocer la diversidad genética caprina en España mediante la caracterización genética de 18 poblaciones caprinas autóctonas españolas con microsatélites.

\section{MATERIAL Y MÉTODOS}

En la tabla I se presentan las razas y el número de animales muestreados en cada una de ellas. Además de las 15 razas caprinas oficialmente reconocidas, se han incluido en el estudio, dos poblaciones insulares peculiares, una asilvestrada, Ajuí de Fuerteventura y otra recientemente definida, cabra de Formentera. Se han analizado también animales de los dos ecotipos de la raza Tinerfeña: Tinerfeña Norte y Tinerfeña Sur. El ADN de las muestras se ha extraído mediante el Kit BLOODCLEAN de Purificación de ADN (BIOTOOLS - Biotechnological \& Medical Laboratories, S.A. Madrid, España) siguiendo las indicaciones del fabricante para este kit. Se han amplificado 23 microsatélites (BM1329, BM1818, BM6506, BM6526, BM8125, CSRD247, CRSM60, CSSM66, ETH10, ETH22, HSC, ILSTS011, INRA063, MAF65, MAF209, McM527, MM12, OarFCB 11, OarFCB48, OarFCB304, SRCRSP8, SPS1 15, TGLA122) mediante la técnica de la reacción en cadena de la polimerasa (PCR) según la metodología de Martínez et al. (2000). Los diferentes alelos han sido visualizados mediante electroforesis en un secuenciador automático ABI377XL (Applied Biosystems, Foster City, CA, USA). El análisis de los fragmentos y la tipificación alélica se realiza mediante los programas informáticos Genescan Analysis ${ }^{\circledR} 3.1 .2$ y Genotyper ${ }^{\circledR} 2.5 .2$ respectivamente. Se han calculado las frecuencias alélicas, el número medio de alelos, las Heterocigosis Esperada y Observada mediante el programa Microsatellite Toolkit (Park, 2001). Los valores de $\mathrm{F}_{\text {IS }}$ con un intervalo de confianza del $95 \%$ se han calculado con el programa informático Genetix v. 4.02 (Belkhir et al., 2003) y la prueba de equilibrio Hardy-Weinberg (HW) mediante el programa Genepop v. 3.1c (Raymond y Rousset, 1995), que aplica el test exacto de Fisher usando el método en cadena de Monte Carlo Markov (Guo y Thompson, 1992).

\section{RESULTADOSYDISCUSIÓN}

Aunque algunas de las razas incluidas en este trabajo han sido previamente caracterizadas genéticamente (Martínez et al., 2004; Acosta et al., 2005; Cañón et al., 2006; Martínez et al., 2006-2007; Delgado et al., 2009), es la primera vez que se presentan datos de la diversidad genética de otras razas como es el caso de la raza Ibicenca o la cabra de Formentera, entre otras. Este estudio es el más amplio en cuanto a caracterización genética de razas caprinas autóctonas españolas ya que se estudian 15 de las 22 razas reconocidas oficialmente más dos poblaciones asilvestradas. Los 23 microsatélites empleados resultan polimórficos en todas las poblaciones estudiadas excepto el locus ETH225, que ha sido monomórfico en la Blanca de Rasquera y en la cabra de Formentera. La cabra de Formentera es la que ha mostrado un número medio de alelos más bajo (tabla I), pero este valor podría deberse al escaso número de muestras analizadas $(\mathrm{N}=11)$. La cabra 


\section{BIODIVERSIDAD CAPRINA EN ESPAÑA}

Palmera es la que ha resultado tener los menores valores tanto de número medio de alelos como de heterocigosis esperada y observada mientras que los valores más altos se han encontrado en la raza Florida (NMA y He) y en la Retinta (Ho). Los valores de NMA, He y Ho promedio para todas las razas es de 7,04, 0,668 y 0,621, estos valores son similares a los encontrados en razas caprinas portuguesas (Bruno-deSousa et al., 2010), en razas europeas o de Oriente Medio (Cañón et al., 2006) o en razas chinas (Li et al., 2002). Once de las 18 poblaciones muestran un exceso de homocigosis con valores de $\mathrm{F}_{\text {IS }}$ significativamente diferentes de 0 (tabla I), siendo las razas
Mallorquina e Ibicenca las que muestran los mayores valores de $\mathrm{F}_{\text {IS }}$. También son estas razas las que presentan un mayor número de marcadores desviados significativamente del equilibrio Hardy-Weinberg.

En este trabajo se presenta la caracterización genética de poblaciones autóctonas caprinas explotadas en la mayor parte del territorio español con el objetivo de obtener una visión general del mapa de la diversidad genética caprina en España. Aunque sería interesante completar este mapa con razas del noroeste y del centro de la península Ibérica, a la vista de los resultados obtenidos se puede concluir que en España existe una gran diversidad genética en la especie

Tabla I. Número de muestras analizadas (N), número promedio de alelos (NMA), heterocigosidad media esperada (He), heterocigosidad media observada $(\mathrm{Ho})$ y sus respectivas desviaciones estándar (DE), valores de Fis con sus intervalos de confianza (IC) y número de loci desviados del equilibrio Hardy-Weinberg $(H W E d)(p<0,05)$ calculados con 23 microsatélites en 18 poblaciones caprinas españolas. (Number of samples analysed $(\mathrm{N})$, average number of alleles (NMA), average expected heterozygosity (He), average observed heterozygosity $(\mathrm{Ho})$ and their standard (DE) deviations, inbreeding coefficient (Fis) with their confidence interval (IC) and number of loci with deviations from Hardy-Weinberg equilibrium (HWEd) $(p<0.05)$ estimated with 23 microsatellites in 18 Spanish goat populations).

\begin{tabular}{|c|c|c|c|c|c|c|c|}
\hline Población & $\mathrm{N}$ & NMA (DE) & $\mathrm{He}(\mathrm{DE})$ & Ho (DE) & & Fis (IC) & HWEd \\
\hline Blanca Andaluza & 40 & $7,30(2,91)$ & $0,692(0,038)$ & $0,643(0,016)$ & 0,072 & $(0,027-0,089)^{*}$ & * 1 \\
\hline Blanca Celtibérica & 40 & $7,91 \quad(3,37)$ & $0,693(0,040)$ & $0,639(0,016)$ & 0,080 & $(0,029-0,103)^{*}$ & 2 \\
\hline Malagueña & 40 & $7,30(3,11)$ & $0,706(0,038)$ & $0,632(0,016)$ & 0,104 & $(0,034-0,146)^{*}$ & 2 \\
\hline Murciano-Granadina & 40 & $7,04(2,51)$ & $0,677(0,045)$ & $0,621(0,016)$ & 0,084 & $(0,024-0,117)^{*}$ & * 3 \\
\hline Florida & 50 & $8,74(4,26)$ & $0,720(0,033)$ & $0,694(0,014)$ & 0,036 & $(-0,003-0,055)$ & ) 1 \\
\hline Payoya & 36 & $7,22(3,38)$ & $0,700(0,038)$ & $0,697(0,016)$ & 0,007 & $(-0,041-0,026)$ & 1 \\
\hline Negra Serrana & 42 & $7,00(2,91)$ & $0,682(0,036)$ & $0,623(0,016)$ & 0,087 & $(0,032-0,115)^{*}$ & * 5 \\
\hline Retinta & 15 & $6,14(2,53)$ & $0,717(0,037)$ & $0,702(0,026)$ & 0,022 & $(-0,093-0,056)$ & ) 0 \\
\hline Azpi-Gorri & 50 & $7,74(3,22)$ & $0,688(0,038)$ & $0,669(0,014)$ & 0,028 & $(-0,022-0,055)$ & 1 \\
\hline Blanca de Rasquera & 57 & $7,52(3,40)$ & $0,662(0,045)$ & $0,588(0,014)$ & 0,114 & $(0,068-0,139)^{*}$ & * 3 \\
\hline Cabra de Formentera & 11 & $4,32(1,59)$ & $0,610(0,047)$ & $0,558(0,032)$ & 0,088 & $(-0,074-0,134)$ & 0 \\
\hline Ibicenca & 78 & $8,00(3,34)$ & $0,678(0,040)$ & $0,582(0,012)$ & 0,142 & $(0,094-0,177)^{*}$ & * 6 \\
\hline Mallorquina & 58 & $8,22(2,66)$ & $0,687(0,034)$ & $0,595(0,014)$ & 0,134 & $(0,084-0,165)^{*}$ & * 7 \\
\hline Ajuí & 55 & $7,13(2,51)$ & $0,681(0,025)$ & $0,631(0,014)$ & 0,073 & $(0,025-0,101)^{*}$ & 3 \\
\hline Majorera & 85 & $7,74(2,96)$ & $0,660(0,030)$ & $0,604(0,012)$ & 0,085 & $(0,044-0,112)^{*}$ & * 6 \\
\hline Palmera & 47 & $4,70(1,89)$ & $0,524(0,039)$ & $0,515(0,016)$ & 0,018 & $(-0,048-0,053)$ & ) 3 \\
\hline Tinerfeña Norte & 53 & $5,87(2,46)$ & $0,624(0,034)$ & $0,580(0,015)$ & 0,072 & $(0,015-0,104)^{*}$ & * 4 \\
\hline Tinerfeña Sur & 70 & $6,91(2,91)$ & $0,628(0,035)$ & $0,608(0,013)$ & 0,033 & $(-0,006-0,055)$ & ) 1 \\
\hline Promedio & & $7,04 \pm 1,15$ & $0,668 \pm 0,047$ & $0,621 \pm 0,050$ & \multicolumn{2}{|c|}{$0,071 \pm 0,040$} & $5,5 \pm 3,05$ \\
\hline
\end{tabular}




\section{MARTÍNEZ ETAL.}

caprina. Análisis adicionales para determinar la estructura genética y las relaciones genéticas entre las poblaciones caprinas estudiadas podrían ayudar a ampliar el conocimiento del estado de estas razas, sobre todo de aquellas que se encuentran más amenazadas, que podría ser de gran utilidad a la hora de abordar actuaciones de conservación o de mejora de las razas caprinas españolas.

\section{BIBLIOGRAFÍA}

Acosta, J.M., Martínez, A., Pestano, J., Cabello, A., Brown, R.P., Sarah-Rey, S. y Delgado, J.V. 2005. Caracterización genética de la cabra Majorera de Fuerteventura con microsatélites. Arch. Zootec., 54: 261-266.

Belkhir, K., Borsa, P., Chikhi, L., Raufaste, N. et Bonhomme, F. 2003. Genetix: 4.05 Logiciel sous WindowsTM pour la genetique des populations. Université de Montpellier. Montpellier. France.

Bruno-de-Sousa, C., Martinez, A.M., Ginja, C., Santos-Silva, F., Carolino, M.I., Delgado, J.V. and Gama, L.T. 2010. Genetic diversity and population structure in Portuguese goat breeds. Livest. Sci., doi:10.1016/j.livsci.2010.06.159.

Cañón, J., García, D., García-Atance, M.A., Obexer-Ruff, G., Lenstra, P., Ajmone-Marsan, P., Dunner, S. and Consortium, E. 2006. Geographical partitioning of goat diversity in Europe and the Middle East. Anim. Genet., 37: 327-334.

Delgado, J.V., León, J.M., Martínez, A.M., Pleguezuelos, J. y Barba, C. 2009. Control genealógico en la raza Murciano-Granadina mediante el uso de microsatélites del ADN. Arch. Zootec., 58 (Supl. 1): 485-489.

Guo, S.W. and Thompson, E.A. 1992. Performing the exact test of Hardy-Weinberg proportions for multiple alleles. Biometrics, 48: 361-372.

Li, M., Zhao, S., Bian, C., Wang, H., Wei, H., Liu, B., Yu, M., Fana, B., Chen, L., Zhu, M., Li, S., Xiong,

\section{AGRADECIMIENTOS}

Los análisis de este trabajo han sido financiados por los proyecto de conservación de recursos zoogenéticos concedidos por el INIA RZ2004-00037-00-00, RZ200600005-C02-00, RZ2007-00005-C02-01 y RZ2007-00005-C02-02, todos ellos cofinanciados con fondos europeos.

T. and Li, K. 2002. Genetic relationships among twelve Chinese indigenous goat populations based on microsatellite analysis. Genet. Select. Evol., 34: 729-44.

Martínez, A.M., Delgado, J.V., Rodero, A. and Vega-Pla, J.L. 2000. Genetic structure of the Iberian pig breed using microsatellites. Anim. Genet., 31: 295-301.

Martínez, A.M., Carrera, M.P., Acosta, J.M., Rodríguez-Gallardo, P.P., Cabello, A., Camacho, E. and Delgado, J.V. 2004. Genetic characterisation of the Blanca Andaluza goat based on microsatellite markers. S. Afr. J. Anim. Sci., 34 (Suppl. 1): 17-19.

Martínez, A.M., Acosta, J., Vega-Pla, J.L. and Delgado, J.V. 2006. Analysis of the genetic structure of the canary goat populations using microsatellites. Livest. Sci., 102: 140-145.

Martínez, A.M., Rocha, L., Quiroz, J. y Delgado, J.V. 2007. Estudio de la diversidad genética intrarracial de la cabra Murciano-Granadina con microsatélites de ADN. Arch. Zootec., 56 (Supl. 1): 417-420.

Park, S.D.E. 2001. Trypanotolerance in West African Cattle and the population genetic effects of selection. University of Dublin. Dublin.

Raymond, M. and Rousset, F. 1995. GENEPOP (Version 1.2): Population genetics software for exact test and ecumenicism. J. Hered., 86: 248-249. 